\title{
Study of nonclassicality in fifth harmonic generation nonlinear optical process
}

\author{
Priyanka, Savita Gill* \\ Department of Applied Science, University Institute of Engineering and Technology, \\ Kurukshetra 136119, India \\ Chauhan7101@gmail.com, *Savita2015@kuk.ac.in
}

DOI 10.17586/2220-8054-2021-12-1-65-72

\begin{abstract}
We have examined non classical effect i.e. higher order single mode antibunching and intermodel antibunching and higher order sub-poissonian photon statistics (HOSPS) in fifth harmonic generation non linear optical process using short time interaction technique. We have found that nonclassical effects directly depend on number of photons prior to interaction with non linear medium. The higher the number of photons present prior to an interaction, the higher will be the nonclassicality in the system. It is additionally found that stoke mode doesn't fulfill the condition of single mode antibunching and HOSPS in fifth harmonic generation process. To examine the optical nonlinearity of nanoparticles, there are significant research efforts concerning the estimation of higher order nonlinear susceptibility which can be utilized as a source for the generation of higher order harmonic generation nonlinear optical processes [19].
\end{abstract}

Keywords: higher order sub-poissonian photon statistics, single mode and intermodel antibunching, optical processes.

Received: 6 February 2021

Revised: 18 February 2021

\section{Introduction}

It is conceivable to characterize a state as a nonclassical state that does exclude any classical analog [1-3]. A radiation field is supposed to be nonclassical if the Glauber Sudarshan P-function of the field becomes more negative than the delta function. The investigation of nonclassical properties of a designed quantum state is a vital field in quantum state designing and quantum information processesing, since the presence of nonclassical qualities gives quantum supremacy [4,5]. Some notable instances of nonclassical are antibunching and sub-Poissonian photon statistics. Investigation of these nonclassical properties is consistently significant in field of their intriguing applications with regards to field of quantum computing, quantum communication and quantum cryptography [6-10]. All the above actual frameworks are experimentally feasible and are effectively seen at nonlinear optics research centers [11, 12]. After the cooperation, photon statistics can be achieved experimentally using homodyne detection procedure [13,14]. The improvements in nanotechnology and nanoscience have given new freedoms to non linear optics. Over the most recent couple of many years, a number of studies on non linear optical properties of novel materials have been performed due to the capability of these materials in optical equipment applications [15-18]. The most impressive strategy to quantify the higher order susceptibilities is Z-scan technique [19] which can be utilized as a source of generations of higher order harmonic generation non linear optical processes. Numerous different creators have additionally predicted upgraded optical nonlinearities, arising because of quantum confinement impacts, regarding the relating mass materials [20-22]. Generation of third, fifth and seventh harmonics by filamentation of mid-infrared laser pulses in air was as of late tentatively noticed [23-26].

These two nonclassical effects may have lower and higher order renditions, investigation of lower order nonclassicality in a quantum state is accounted for in writing since its origin, yet premium in higher order is generally new and promising from an experimental perspective [27]. Out of these higher order nonclassical impacts, higher order squeezing has been focused on in detail [28-31] yet higher order sub-Poissonian photon statistics (HOSPS) and higher order antibunching (HOA) has not yet been concentrated definitely. The concept of HOA was introduced by Lee [32] and it has been anticipated in a two photon coherent state [32,33,33], and a shadowed negative binomial state [34]. Yet, HOA has all the earmarks of being an extremely uncommon wonder. Recently, Prakash and Mishra have set up broad measure for HOSPS in a real system [35].

Remembering these realities, the current investigation shows that higher order single mode antibunching, intermodel antibunching, and HOSPS can be found in fifth harmonic generation process, and in this process, they appear simultaneously. In this paper, section 2 gives the condition of nonclassicality of the nonlinear optical system. Section 3 will introduce a second order solution of equation of motion of fifth harmonic generation process and show the presence of higher order single mode antibunching, intermodel antibunching and HOSPS and their immediate relationship with pump photons present in the system. In section 6 is commited to conclusion. 


\section{Criteria of nonclassicality of a nonlinear optical system}

\subsection{Criteria for single mode and intermodel antibunching}

HOA is communicated in wording factorial moment of number operator. HOA criteria for single mode was presented by Lee is given as [32]:

$$
R(m, l)=\frac{\left\langle N_{x}^{m-1}\right\rangle\left\langle N_{x}^{l+1}\right\rangle}{\left\langle N_{x}^{m}\right\rangle\left\langle N_{x}^{l}\right\rangle}-1<0,
$$

where $N$ is numerical operator. $\left\langle N^{(k)}\right\rangle=\langle N(N-1)(N-2) \ldots \ldots .(N-k+1)\rangle$ is the $k^{\text {th }}$ factorial moment of number operator. Integers $l$ and $m$ fulfilling condition $l \leq m \leq 1$ and $x$ subscript denotes specific mode. $m=1$ is choosen by Ba An [33] and criteria of $l^{\text {th }}$ order antibunching is reduced to:

$$
\left\langle N_{x}^{l+1}\right\rangle<\left\langle N_{x}^{l}\right\rangle\left\langle N_{x}\right\rangle
$$

by streamlining equation (2) and acquire condition of $l^{\text {th }}$ order antibunching as

$$
d(l)=\left\langle N_{x}^{l+1}\right\rangle-\left\langle N_{x}\right\rangle^{l+1}<0
$$

furthermore, intermodel antibunching criteria is given as:

$$
D_{a b}=\left(\Delta N_{a b}\right)^{2}=\left\langle a^{\dagger} a b^{\dagger} b\right\rangle-\left\langle a^{\dagger} a\right\rangle\left\langle b^{\dagger} b\right\rangle<0,
$$

where equation (3) gives us the condition to single mode antibunching and equation (4) gives us the conditions for intermodel antibunching. Accordingly, we can say that single photon source utilized in quantum cryptography ought to fulfill the criteria given in equation (3) of HOA [36].

\subsection{Condition for Higher Order sub-Poissonian Photon Statistics (HOSPS)}

Prakash and Mishra [35] provides condition of $(l-1)^{t h}$ order HOSPS is given as:

$$
D(l-1)=\sum_{k=0}^{l} \sum_{i=0}^{l-k}{ }^{l} C_{k}(-1)^{k} S_{2}(l-k, i)\left\langle N^{i}\right\rangle\langle N\rangle^{k}-\sum_{k=0}^{l} \sum_{i=0}^{l-k}{ }^{l} C_{k}(-1)^{k} S_{2}(l-k, i)\langle N\rangle^{k+i}<0,
$$

where $S_{2}(l, k)$ is a Stirling number of the second order. The criteria for second order subpoissonian photon statistics for which $l=3$ is given as:

$$
D(2)=\left\langle N^{3}\right\rangle+2\langle N\rangle^{3}-3\left\langle N^{2}\right\rangle\langle N\rangle+3\left\langle N^{2}\right\rangle-3\langle N\rangle^{2}<0,
$$

equation (6) is the criteria of second order sub-Poissonian photon statistics.

\section{Fifth harmonic generation process}

To examine higher order single mode antibunching, intermodel antibunching and HOSPS, we have picked fifth harmonic generation process so that absorption of five photons, each having frequency $\omega_{1}$ with emission of one photon of frequency $\omega_{2}$ where $\omega_{2}=5 \omega_{1}$. Hamiltonian for the said process is

$$
H=\omega_{1} a^{\dagger} a+\omega_{2} b^{\dagger} b+g\left(a^{5} b^{\dagger}+a^{\dagger 5} b\right),
$$

where $g$ is the coupling constant, $a^{\dagger}(a), b^{\dagger}(b)$ are the creation (annihilation) operators, respectively. $A=a \exp i \omega_{1} t$, $B=b \exp i \omega_{2} t$ are the slowly varying operators at frequencies $\omega_{1}$ and $\omega_{2}$.

\subsection{Time evolution of pump mode $A$}

Time evolution of operator in pump mode $\mathrm{A}$ is given by Heisenberg equation of motion as:

$$
\frac{d A}{d t}=\frac{\partial A}{\partial t}+i[H, A]
$$

we obtain:

$$
\dot{A}=-5 i g A^{+4} B
$$

and

$$
\dot{B}=-i g A^{5} \text {. }
$$

Now using the short time approximation technique, expanding $A(t)$ using Taylor's series expansion and taking terms up to $g^{2} t^{2}$ as:

$$
A(t)=A-5 i g t A^{\dagger 4} B+\frac{5}{2} g^{2} t^{2}\left(20 A^{\dagger 3} A^{4} N_{B}+120 A^{\dagger 2} A^{3} N_{B}+240 A^{\dagger} A^{2} N_{B}+120 A N_{B}-A^{\dagger 4} A^{5}\right) .
$$


Using equation (11), the numerical operator $N_{A}(t)=A^{\dagger}(t) A(t)$ is given as:

$$
\begin{gathered}
N_{A}(t)=A^{\dagger} A-5 i g t\left(A^{\dagger 5} B-A^{5} B^{\dagger}\right)+5 g^{2} t^{2}\left(25 A^{\dagger 4} A^{4} N_{B}+200 A^{\dagger 3} A^{3} N_{B}+\right. \\
\left.+600 A^{\dagger 2} A^{2} N_{B}+600 A^{\dagger} A N_{B}+120 N_{B}-A^{\dagger 5} A^{5}\right)
\end{gathered}
$$

To study antibunching, initially we assume a quantum state which is the product of coherent state $|\alpha\rangle$ for pump mode A and vacuum state $|0\rangle$ for stokes mode B i.e.

$$
|\psi\rangle=|\alpha\rangle_{A}|0\rangle_{B}
$$

Using equation (13) in equation (12), we get expectation value of $\left\langle N_{A}(t)\right\rangle_{\alpha}$ is given as:

$$
\left\langle N_{A}(t)\right\rangle_{\alpha}=|\alpha|^{2}-5 g^{2} t^{2}|\alpha|^{10},
$$

where $A|\alpha\rangle=\alpha|\alpha\rangle$. By using straight forward description we can get:

$$
\left\langle N_{A}^{2}(t)\right\rangle_{\alpha}=\left\langle A^{\dagger 2}(t) A^{2}(t)\right\rangle=|\alpha|^{4}-10 g^{2} t^{2}\left(|\alpha|^{12}+2|\alpha|^{10}\right),
$$

and

$$
\left\langle N_{A}^{3}(t)\right\rangle_{\alpha}=|\alpha|^{6}-5 g^{2} t^{2}\left(3|\alpha|^{14}+12|\alpha|^{12}+12|\alpha|^{10}\right)
$$

and

$$
\left\langle N_{A}^{4}(t)\right\rangle_{\alpha}=|\alpha|^{8}-20 g^{2} t^{2}\left(|\alpha|^{16}+6|\alpha|^{14}+12|\alpha|^{12}+24|\alpha|^{10}\right),
$$

and

$$
\left\langle N_{A}^{5}(t)\right\rangle_{\alpha}=|\alpha|^{10}-5 g^{2} t^{2}\left(5|\alpha|^{18}+40|\alpha|^{16}+120|\alpha|^{14}+120|\alpha|^{12}+24|\alpha|^{10}\right),
$$

now using equations (14-18) in equation (3), we get:

$$
d_{A}(1)_{\alpha}=-20 g^{2} t^{2}|\alpha|^{10},
$$

and

and

$$
d_{A}(2)_{\alpha}=-60 g^{2} t^{2}\left(|\alpha|^{12}+|\alpha|^{10}\right)
$$

$$
d_{A}(3)_{\alpha}=-120 g^{2} t^{2}\left(|\alpha|^{14}+2|\alpha|^{12}+|\alpha|^{10}\right)
$$

and

$$
d_{A}(4)_{\alpha}=-40 g^{2} t^{2}\left(5|\alpha|^{16}+15|\alpha|^{14}+15|\alpha|^{12}+3|\alpha|^{10}\right) .
$$

Equations (19-22) show that the fifth harmonic generation process satisfies the criterian for normal and higher order antibunching.

To study higher order antibunching, we have taken the initial state which is the product of vacuum state $|0\rangle$ for pump mode $\mathrm{A}$ and $|\beta\rangle$ for stokes mode $\mathrm{B}$ i.e.:

$$
|\psi\rangle=|0\rangle_{A}|\beta\rangle_{B}
$$

Now taking expectation values of $N_{A}(t), N_{A}^{2}(t), N_{A}^{3}(t), N_{A}^{4}(t)$ and $N_{A}^{5}(t)$ in pump mode A, with respect to condition (23), is given as:

$$
\begin{gathered}
\left\langle N_{A}(t)\right\rangle_{\beta}=600 g^{2} t^{2}|\beta|^{2}, \\
\left\langle N_{A}^{2}(t)\right\rangle_{\beta}=2400 g^{2} t^{2}|\beta|^{2}, \\
\left\langle N_{A}^{3}(t)\right\rangle_{\beta}=7200 g^{2} t^{2}|\beta|^{2}, \\
\left\langle N_{A}^{4}(t)\right\rangle_{\beta}=14400 g^{2} t^{2}|\beta|_{(27)}^{2}, \\
\left\langle N_{A}^{5}(t)\right\rangle_{\beta}=14400 g^{2} t^{2}|\beta|^{2} .
\end{gathered}
$$

Using equations (24-28) in equation (3), we get normal and higher order antibunching in pump mode A with respect to $|0\rangle|\beta\rangle$ is given as:

$$
\begin{gathered}
d_{A}(1)_{\beta}=2400 g^{2} t^{2}|\beta|^{2}, \\
d_{A}(2)_{\beta}=7200 g^{2} t^{2}|\beta|^{2}, \\
d_{A}(3)_{\beta}=14400 g^{2} t^{2}|\beta|^{2}, \\
d_{A}(4)_{\beta}=14400 g^{2} t^{2}|\beta|^{2} .
\end{gathered}
$$

Equations (29-32) show that neither normal antibunching nor higher order antibunching is present in pump mode A with respect to the quantum state $|0\rangle|\beta\rangle$.

Now using equations $(12,13$ and 23$)$ in equation (6), we get:

$$
D(2)_{\alpha}=-120 g^{2} t^{2}|\alpha|^{10}
$$




$$
D(2)_{\beta}=0 .
$$

We obtain a negative value in equation (33) with respect to the quantum state $|\psi\rangle=|\alpha\rangle_{A}|0\rangle_{B}$, which shows the higher order non-classicality, i.e. HOSPS, exists in pump mode A.

\subsection{Time evolution of stoke mode B:}

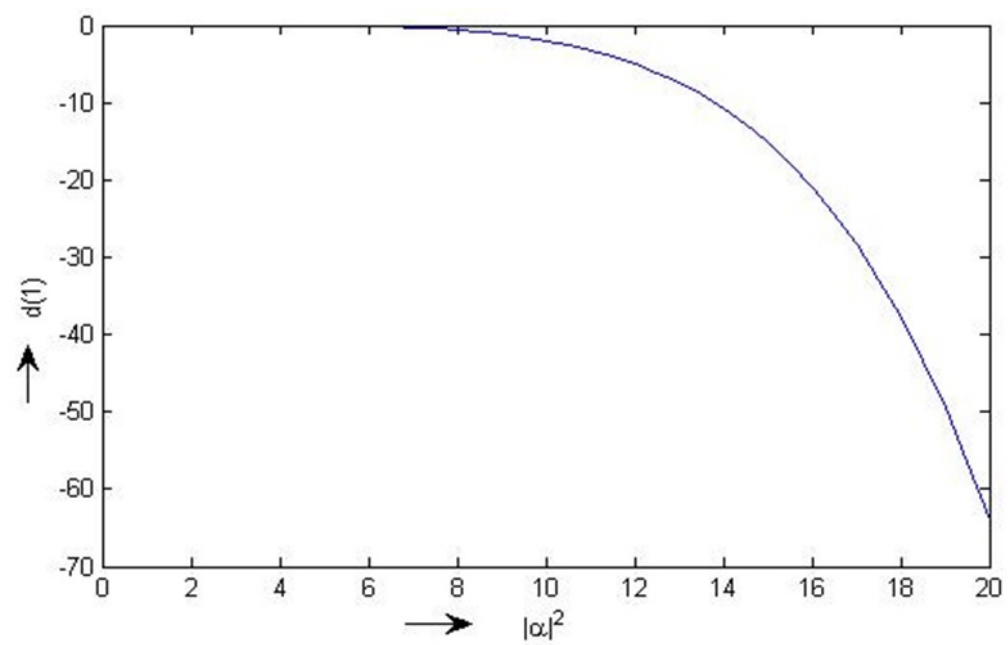

FIG. 1. Variation of normal antibunching $d(1)$ with $|\alpha|^{2}$ in fifth harmonic generation process (taking $g^{2} t^{2} \approx 10^{-6}$ )

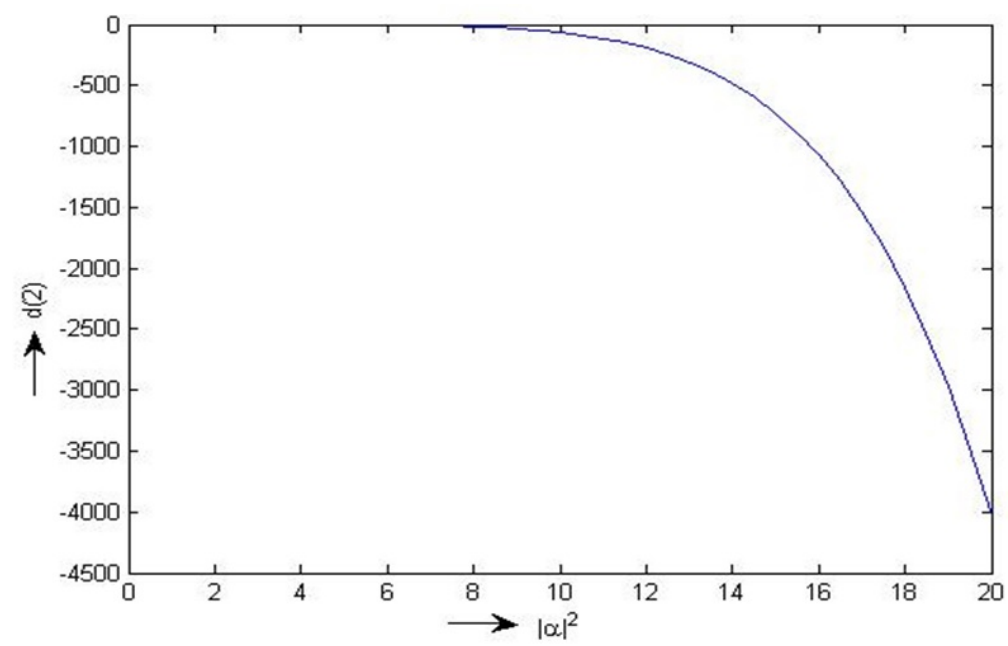

FIG. 2. Variation of second order antibunching $d(2)$ with $|\alpha|^{2}$ in fifth harmonic generation process (taking $g^{2} t^{2} \approx 10^{-6}$ )

Using Heisenberg equation of motion, we get:

$$
\dot{A}=-5 i g A^{+4} B, \quad \dot{B}=-i g A^{5} .
$$

Using Taylor's series expansion, time evolution of $\mathrm{B}$ operator is given as:

$$
B(t)=B-i g t A^{5}-\frac{5}{2} g^{2} t^{2}\left[5 A^{\dagger 4} A^{4} B+40 A^{\dagger 3} A^{3} B+120 A^{\dagger 2} A^{2} B+120 A^{\dagger} A B+24 B\right],
$$

where $N_{A}=A^{+} A, N_{B}=B^{+} B$ and $N_{C}=C^{+} C$.

The number operator in $\mathrm{B}$ mode is given as:

$$
N_{B}(t)=B^{\dagger}(t) B(t)
$$




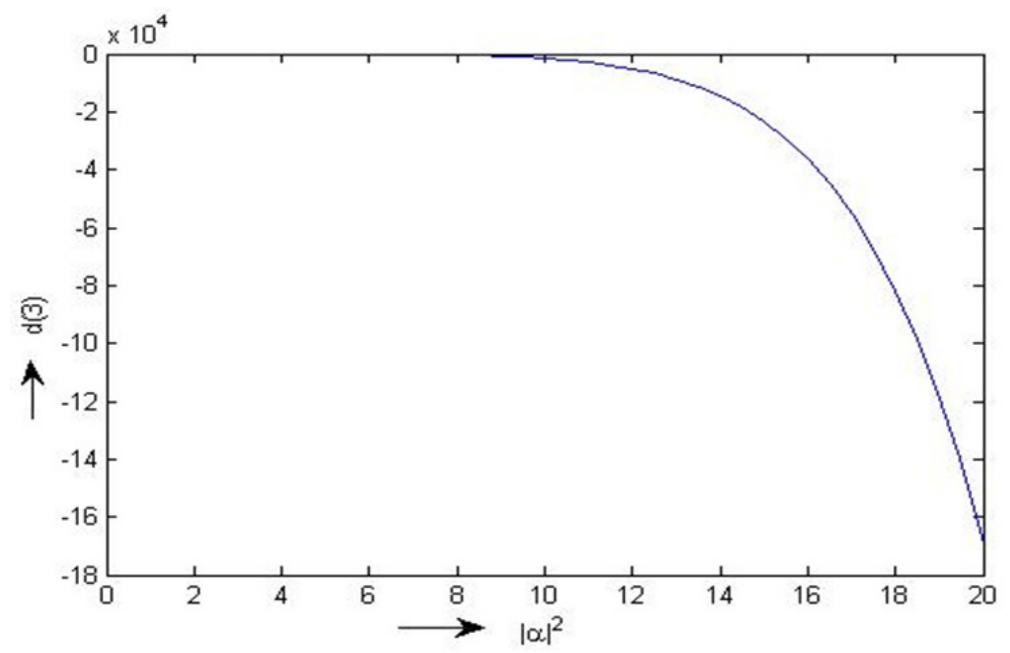

FIG. 3. Variation of third order antibunching $d(3)$ with $|\alpha|^{2}$ in fifth harmonic generation process (taking $g^{2} t^{2} \approx 10^{-6}$ )

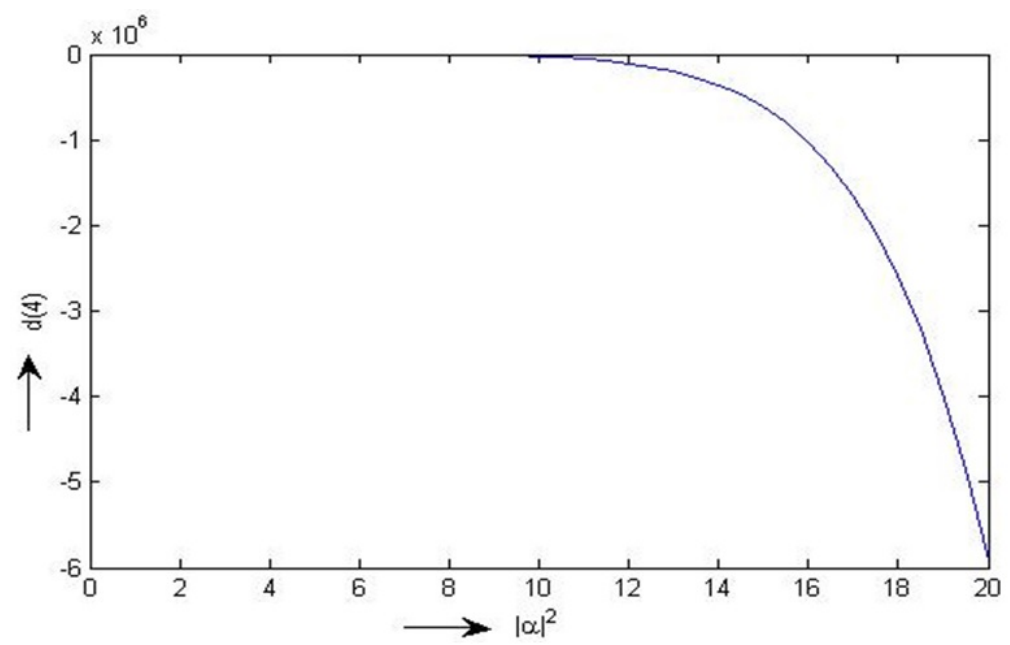

FIG. 4. Variation of fourth order antibunching $d(4)$ with $|\alpha|^{2}$ in fifth harmonic generation process (taking $g^{2} t^{2} \approx 10^{-6}$ )

$$
\begin{gathered}
N_{B}(t)=B^{\dagger} B-i g t\left(A^{5} B^{\dagger}-A^{\dagger 5} B\right)-5 g^{2} t^{2}\left(5 A^{\dagger 4} A^{4} N_{B}+40 A^{\dagger 3} A^{3} N_{B}+120 A^{\dagger 2} A^{2} N_{B}+\right. \\
\left.+120 A^{\dagger} A N_{B}+24 N_{B}\right)+g^{2} t^{2} A^{\dagger 5} A^{5} .
\end{gathered}
$$

The expectation value of $N_{B}(t)$ with respect to the initial condition $|\alpha\rangle|0\rangle$ is given as:

$$
\left\langle N_{B}(t)\right\rangle_{\alpha}=g^{2} t^{2}|\alpha|^{10} \text {. }
$$

Now, the expectation value of $N_{B}^{2}(t), N_{B}^{3}(t), N_{B}^{4}(t)$ and $N_{B}^{5}(t)$ is given as:

$$
\left\langle N_{B}^{2}(t)\right\rangle_{\alpha}=0
$$

and

and

$$
\left\langle N_{B}^{3}(t)\right\rangle_{\alpha}=0
$$

and

$$
\left\langle N_{B}^{4}(t)\right\rangle_{\alpha}=0
$$

$$
\left\langle N_{B}^{5}(t)\right\rangle_{\alpha}=0 .
$$

Now, using equations (38-42) in equation (3), we get:

$$
d_{B}(1)_{\alpha}=0,
$$




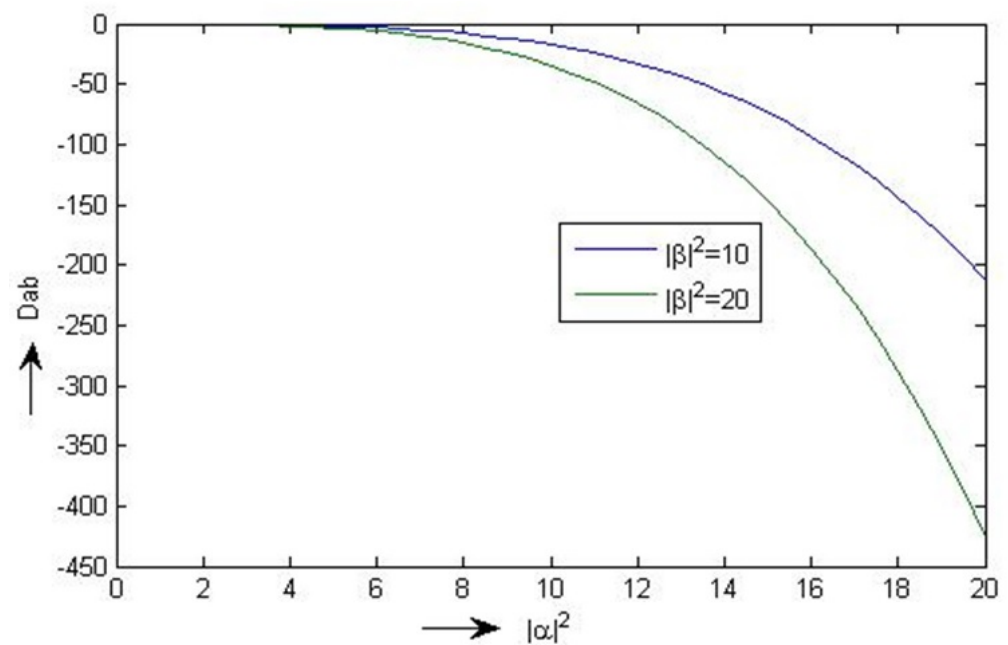

FIG. 5. Variation of intermodel antibunching $D_{a b}$ with $|\alpha|^{2}$ in fifth harmonic generation process (taking $g^{2} t^{2} \approx 10^{-6}$ )

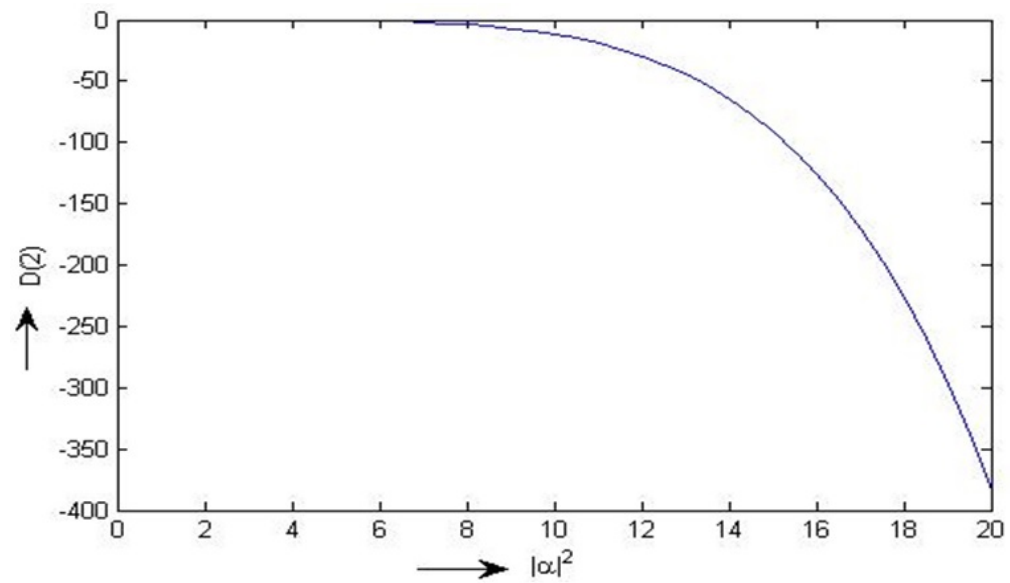

FIG. 6. Variation of higher order sub-Poissonian photon statistics $D(2)$ with $|\alpha|^{2}$ in fifth harmonic generation process (taking $g^{2} t^{2} \approx 10^{-6}$ )

$$
\begin{aligned}
& d_{B}(2)_{\alpha}=0, \\
& d_{B}(3)_{\alpha}=0, \\
& d_{B}(4)_{\alpha}=0 .
\end{aligned}
$$

using initial condition (23), we obtain expectation values of $N_{B}(t), N_{B}^{2}(t), N_{B}^{3}(t), N_{B}^{4}(t)$ and $N_{B}^{5}(t)$ in stoke mode $\mathrm{B}$ is given as:

$$
\begin{aligned}
& \left\langle N_{B}(t)\right\rangle_{\beta}=|\beta|^{2}-120 g^{2} t^{2}|\beta|^{2}, \\
& \left\langle N_{B}^{2}(t)\right\rangle_{\beta}=|\beta|^{4}-240 g^{2} t^{2}|\beta|^{4}, \\
& \left\langle N_{B}^{3}(t)\right\rangle_{\beta}=|\beta|^{6}-360 g^{2} t^{2}|\beta|^{6}, \\
& \left\langle N_{B}^{4}(t)\right\rangle_{\beta}=|\beta|^{8}-480 g^{2} t^{2}|\beta|^{8}, \\
& \left\langle N_{B}^{5}(t)\right\rangle_{\beta}=|\beta|^{10}-600 g^{2} t^{2}|\beta|^{10},
\end{aligned}
$$

using equations (47-51) in equation (3), we obtain the normal and higher order antibunching in B mode with respect to $|0\rangle|\beta\rangle$ is given as:

$$
\begin{aligned}
& d_{B}(1)_{\beta}=0, \\
& d_{B}(2)_{\beta}=0, \\
& d_{B}(3)_{\beta}=0,
\end{aligned}
$$




$$
d_{B}(4)_{\beta}=0 .
$$

Equations (43-46) and (52-55) show that neither normal antibunching nor higher order antibunching is present in stoke mode $\mathrm{B}$ with respect to quantum states $|\alpha\rangle|0\rangle$ and $|0\rangle|\beta\rangle$ respectively.

Now, using equations (13, 23 and 37) in equation (6), we get:

$$
\begin{aligned}
& D(2)_{\alpha}=0, \\
& D(2)_{\beta}=0 .
\end{aligned}
$$

Equations (56-57) show that higher order non-classicality i.e. HOSPS is absent in stoke mode B.

Further, using the conditions for intermodel antibunching described in equation (4) and using equations (12) and (37), we obtain following closed expression of $D_{a b}$ :

$$
D_{a b}=-100 g^{2} t^{2}\left(|\alpha|^{2}|\beta|^{2}\right)\left[|\alpha|^{6}+6|\alpha|^{4}+12|\alpha|^{2}+6\right] .
$$

Equation (58) show that intermodel antibunching exist in fifth harmonic generation non linear optical process.

\section{Results}

The presence of single mode and intermodel antibunching and HOSPS in fifth harmonic generation nonlinear process appeared in equations $(19,20,21,22,58,33)$ respectively. On the off chance that we plot a graph between HOA such as $d(1), d(2), d(3), d(4)$, intermodel antibunching say $D_{a b}$ and HOSPS say $D(2)$ with photon number in pump mode A i.e. $|\alpha|^{2}$ respectively, it is clear that higher order single mode antibunching, intermodel antibunching and HOSPS increase non linearly with an increase in $|\alpha|^{2}$. Further, it is obvious from the figures that nonclassicality increases as we go towards the higher order of antibuncing.

\section{Conclusion}

We have revealed normal as well as higher order non-classicality with regards to single mode antibunching and intermodel antibunching and higher order sub-Poissonian photon statistics (HOSPS) in pump mode in fifth harmonic generation nonlinear optical process. HOA as well as HOSPS isn't seen in stoke mode in fifth harmonic generation nonlinear optical process. Along these lines, from the results, we can presume that the higher the number of photons present prior to interaction, the higher will be the nonclassicality in the system.

\section{References}

[1] Meystre P., Sargent M. Elements of Quantum Optics, 2nd edn, Berlin, Springer, 1991.

[2] Dodonov V.V. Nonclassical states in quantum optics: a 'squeezed' review of the first 75 years. Journal of Optics B: Quantum and Semiclassical Optics, 2002, 4.

[3] Hanbury Brown R., Twiss R. Q. Correlation between photons in two coherent beams of light. Nature, 1956, 177(4497), P. 27-29.

[4] Harrow Aram W., Montanaro A. Quantum computational supremacy. Nature, 2017, 549(7671), P. $203-209$.

[5] Neill C., Roushan P., Kechedzhi K., Boixo S., Isakov S.V., Smelyanskiy V., Megrant A., Chiaro B., Dunsworth A., Arya K. A blueprint for demonstrating quantum supremacy with superconducting qubits. Science, 2018, 360(6385), P. 195-199.

[6] Abbott B. P., Abbott R., Abbott T. D., Abernathy M. R., Acernese F., Ackley K., Adams C., Addesso P., Adhikari R. X. et al. Gw151226. Observation of gravitational waves from a 22-Solar mass binary black hole coalescence. Physical Review Letters, 2016, 116(24), P. 241103(114).

[7] Madsen L. S., Usenko V. C., Lassen M., Filip R., Andersen U.L. Continuous variable quantum key distribution with modulated entangled states. Nature communications, 2012, 3(1), P. 1038(1-6).

[8] Bennett C.H. et al. Entanglement- Assisted Classical Capacity of Noisy Quantum Channels. Physical Review Letters, 1993, 83(15), P. 30813084.

[9] Braunstein S.L., Kimble H.J. Dense coding for continous variables. Physical Review A, 2000, 61(4), P. 042302(1-4).

[10] Braunstein et. al. Universal Teleportation with a Twist. Physical Review Letters, 2000, 84(15), P. $3486-3489$.

[11] Bachor H.A. A guide to experiments in quantum optics (Weinheim: Wiley-VCH) chapters 8 and 10.

[12] Vogel W., Welsch D., Wallentowitz S. Quantum optics: an introduction 2nd edition. Berlin, Wiley-VCH, chapter 6.

[13] Erenso D., Vyas R, Singh S. Higher-order sub-Poissonian photon statistics in terms of factorial moments. Journal of Optical Society B, 2002, 19(6), P. 1471-1475.

[14] Vyas R., Singh S. Photon-counting statistics of the degenerate optical parametric oscillator. Physical Review A, 1989, 40(9), 5147-5159.

[15] Kreibig U., Vollmer M. Optical properties of metal clusters. Springer Series in Materials Science, 1995, 25, P. $278-279$.

[16] Seongmin J.U., Wateker P.R., Jeong S. Nonlinear optical properties of zinc doped germane silicate glass optical fibre. Journal of Nonlinear Optical Physics and Materials, 2011, 19(4), P. 791-799.

[17] Xie R.H. Handbook of advanced electronic and photonic materials and devices. H.S. Nalwa (Ed.), Elsevier, 2000,9 , P. $267-307$.

[18] Shuklina A.I., Smirnov A.V., Fedorov B.A., Kirillova S.A., Almjasheva O.V. Structure of nanoparticles in the $\mathrm{ZrO}_{2}-\mathrm{Y}_{2} \mathrm{O}_{3}$ system, as obtained under hydrothermal conditions. Nanosystems: Physics, Chemistry, Mathematics, 2020, 11(6), P. 729-738.

[19] Xie R.H., Rao Q., Ensen L. Encyclopedia of nanoscience and nanotechnology. Nalwa H.S., American Scientific, Los angles, 2003.

[20] Roussignol P.H., Ricard D., Flytzanis C.H.R. Quantum confinement mediated enhancement of optical kerr effect in CdS Se $_{1-x}$ semiconductor microcrystallites. Applied Physics B, 1990, 51(6), P. 437-442. 
[21] Hanamura E. Very large optical nonlinearity of semiconductor microcrystallites. Physical Review B, 1988, 37(3), P. 1273-1279.

[22] Cotter D., Burt M.G., Manning R.J. Below-band-gap third-order optical nonlinearity of Nanometer -size semiconductor crystallites. Physical Review Letters, 1992, 68(8), P. 1200-1203.

[23] Jni J., Yao J., Zeng B., Chu W., Li G., Zhang H., Jing C., Chin S. L., Cheng Y., Xu Z. Comparative investigation of third- and fifth-harmonic generation in atomic and molecular gases driven by midinfrared ultrafast laser pulses. Physics Review A, 2011, 84(5), P. 063846(1-4).

[24] Ariunbold G.O., Polynkin P., Moloney J.V. Third and Fifth harmonic generation by tightly focused femtosecond pulses at $2.2 \mu \mathrm{m}$ wavelength in air. Optics Express, 2012, 20(2), P. 1662-1667.

[25] Kartashov D., Alisauskas S., Pugzlys A., Voronin A.A., Zheltikov A.M., Baltuska A. Third- and fifth-harmonic generation by mid-infrared ultrashort pulses: beyond the fifth- order nonlinearity. Optics Letter, 2012, 37(12), P. 2268-2270.

[26] Nath A., Dharmadhikari J.A., Dharmadhikari A.K., Mathur D. Seventh harmonic generation from tightly focused $2 \mu \mathrm{m}$ ultrashort pulses in air. Optics Letter, 2013, 38(14), P. 2560-2562.

[27] Sperling J., Vogel W., Agarwal G.S. Quantum state engineering by click counting. Physical Review A, 2014, 89(4), P. P. 043829(1-10).

[28] Hong C.K., Mandel L. Higher order squeezing of a quantum field. Physical Review Letters, 1985, 54(4), P. 323-325.

[29] Hong C.K., Mandel L. Generation of higher order squeezing of quantum electromagnetic fields. Physical Review A, 1985, 32(2), P. 974-982.

[30] Hillery M. Amplitude-squared squeezing of the electromagnetic field. Physical Review A, 1987, 36(8), P. 3796-3802.

[31] Priyanka, Gill S. Comparison of non classical effects: quantum phase fluctuation, antibunching and minimum total noise in various non linear optical processes. Nanosystems: Physics, Chemistry, Mathematics, 2020, 11(2), P. 161-170.

[32] Lee C.K. Higher order criteria for nonclassical effects in photon statistics. Physical Review A, 1990, 41(3), P. P. 1721-1723.

[33] An B.N. Multimode higher order antibunching and squeezing in trio coherent states. Journal of Optics B: Quantum and Semiclassical Optics, 2002, 4(3), P. 222-227.

[34] Srinivasan R., Lee C.T. Shadowed negative binomial state. Physical Letter A, 1996, 218(3-6), P. 151-156.

[35] Prakash H., Mishra D.K. Higher order sub-Poissonian photon statistics and their use in detection of Hong and Mandel squeezing and amplitude-squared squeezing. Journal of Physics B: Atomic, Molecular and Optical Physics, 2006, 39(9), P. $2291-2297$.

[36] Pathak A. A mathematical criterion for single photon sources used in quantum cryptography. Indian Journal of Physics, 2006, 80(5), P. 495499. 